\title{
Características clínico-microbiológicas de la meningitis por Streptococcus pneumoniae resistente a la penicilina
}

\author{
Demóstenes Gómez-Barreto, M.C., ${ }^{(1)}$ Ernesto Calderón-Jaimes, M.C ., M.S.P., (2) Romeo S. Rodríguez, M.C., ${ }^{(1)}$ \\ Luz Elena Espinosa de los Monteros, Q .B.P., M. en C., D r. en C., (1) Maricruz Juárez, M.C..1)
}

\begin{abstract}
Gómez-Barreto D, Calderón-Jaimes E, Rodríguez RS, Espinosa de los Monteros LE, Juárez M. Características clínico-microbiológicas de la meningitis por Streptococcus pneumoniae resistente a la penicilina. Salud Publica Mex 1999;41:397-404.
\end{abstract}

\section{Resumen}

Objetivo. Evaluar la susceptibilidad antimicro biana de Streptococcus pneumoniae aislado del líquido cefalorraquídeo de niños con meningitis, así como describir y comparar las características clínicas y microbiológicas, el tratamiento y la evolución del padecimiento entre niños infectados con cepas sensibles y resistentes a la penicilina y la cefalosporina. Material y métodos Treinta y ocho niños con meningitis neumocócica fueron incluidos prospectivamente en el Programa Institucional de Vigilancia de las Infecciones $\mathrm{N}$ eumocócicas, durante el lapso 1994-1998. Los datos clínicos y de laboratorio se colectaron de cada expediente. Resultados Del total de niños, $63 \%$ era menor de dos años de edad, $28.9 \%$ mostró cepas insensibles a la penicilina, $18.4 \%$ tenía resistencia intermedia, y $10.5 \%$ tenía resistencia elevada. El 2.6\% mostró también resistencia a la cefotaxima. La única característica (por la prueba exacta de Fisher) asociada con la resistencia fue: enfermedad de base previa al proceso $(p<0.001)$, y condiciones de gravedad al ingreso como convulsiones, choque tóxico y coma $(p<0.07)$. El curso de la enfermedad y la evolución clínica fue similar para los niños infectados con cepas sensibles a la penicilina 0 a la cefotaxima vs. las cepas no susceptibles. Conclusiones El perfil actual de resistencia del $\mathrm{S}$. pneumoniae a la penicilina y la cefalosporina no se encontró asociado con un aumento en la mortalidad de niños con meningitis neumocócica.

Palabras clave: meningitis neumo cócica; resistencia a penicilina; México
Gómez-Barreto D, Calderón-Jaimes E, Rodríguez RS, Espinosa de los Monteros LE, Juárez M. Streptococcus pneumoniae meningitis resistant to penicillin clinical and microbiological characteristics. Salud Publica Mex 1999;41:397-404.

\begin{abstract}
A bstract
Objective. To evaluate the susceptibility to antibiotics of Streptococcus pneumoniae isolated from cerebrospinal fluid of children with meningitis. To describe and compare the clinical and microbiological characteristics, treatment and outcome among children infected with strains either susceptible or resistant to penicillin and cephalosporin. Material and methods A total of 38 children with pneumococcal meningitis were prospectively enrolled in the Institutional Surveillance Program for Pneumococcal Infections during 1994-1998. Clinical and laboratory data were collected by chart review. Results $0 f$ the 38 children, $24(63 \%)$ were less than 2 years of age, 11 (28.9\%) had drug-resistant $\mathrm{S}$. pneumoniae, $18.4 \%$ had intermediate resistance, $10.5 \%$ high level resistance and $2.6 \%$ also showed high level resistance to cefo taxime.The only associated factors (by Fisher's exact test) associated to resistance were: previous use of antibiotics $(p=0.2)$, underlying disease $(p<0.001)$. Course of illness and clinical course were similar for children infected with penicillin or cefo taxime susceptible, vs. non-susceptible strains. Conclusions Current levels of $S$. pneumoniae resistance to penicillin and cephalosporin are not associated to an increase in mortality in children with meningitis.
\end{abstract}

Key words: pneumococcal meningitis; penicillin resistance; Mexico

(1) Departamento de Infectología, Hospital Infantil de México Federico Gómez, México.

(2) División de Medicina Experimental, Instituto $\mathrm{N}$ acional de Pediatría, México.

Fecha de recibido: 12 de marzo de 1999 - Fecha de aprobado: 12 de julio de 1999 Solicitud de sobretiros: Dr. Demóstenes Gómez Barreto. Hospital Infantil de México Federico Gómez. Dr. Márquez 162, colonia D octores, Delegación Cuauhtémoc, 06720 México, D.F., México. Correo electrónico:dgbarreto30@ hotmail.com 
$\mathrm{E}$ 1 Streptococcus pneumoniae es el agente bacteriano más frecuente en la etiología de las infecciones respiratorias como la otitis media aguda, la sinusitis aguda, la nasofaringoconjuntivitis febril y la neumonía adquirida en la comunidad; asimismo, es etiología de procesos sistémicos invasivos como la bacteriemia sin foco evidente de infección, la neumonía grave y la septicemia con o sin componente meningoencefálico. ${ }^{1-4}$

El tratamiento habitual de todas las infecciones del S. pneumoniae, usualmente tenía como fármaco de selección primaria a la penicilina; sin embargo, la emergencia de la resistencia bacteriana ${ }^{5,6}$ ha cambiado ese concepto, lo que a su vez ha permitido identificar varios factores de riesgo, así como analizar los diversos mecanismos de la resistencia antimicrobiana. ${ }^{7}$

En vista de que la resistencia bacteriana del S. pneumoniae continúa y aumenta, es necesario modificar los diferentes aspectos del tratamiento de acuerdo con esos cambios, aun cuando en la actualidad no hay un consenso respecto al tratamiento de las infecciones invasivas, en particular de aquellas que se presentan fuera del sistema nervioso.

Las estrategias de tratamiento de las infecciones sistémicas producidas por el S. pneumoniae resistente o multirresistente a los betalactámicos, se basan en la actividad bacteriana in vitro, en la observada entre animales de experimentación y en informes de casos o series de casos. La interpretación de las fallas del tratamiento antimicrobiano, como es lógico esperar, es muy complicada, sobre todo cuando existen, entre otros, padecimientos de base que propician la infección neumocócica, lo que no permite valorar la evolución clínica debida sólo al neumococo o modificada por el padecimiento subyacente.

Diversos informes ${ }^{8-11}$ sugieren que el tratamiento con penicilina es inapropiado para la meningitis neumocócica, sobre todo cuando se miden las concentraciones mínimas inhibitorias alcanzadas en el líquido cefalorraquídeo (LCR) para diversos betalactámicos. ${ }^{7,12}$

La presencia de cepas de neumococo resistente a las cefalosporinas de tercera generación, complica aún más la selección del esquema apropiado de tratamiento de las infecciones sistémicas con componente meningoencefálico, por lo cual es necesario analizar el comportamiento clínico de pacientes que padecen meningitis con cepas resistentes a cefalosporinas y a otros fármacos utilizados en el tratamiento empírico inicial.

El propósito de este trabajo, basado en datos del sistema institucional de vigilancia del desarrollo de resistencia del S. pneumoniae en el Hospital Infantil de México Federico Gómez, es evaluar la susceptibi- lidad antimicrobiana de S. pneumoniae aislados del LCR de niños con meningitis; asimismo, se pretende describir y comparar las características clínicas y microbiológicas, el tratamiento y la evolución del padecimiento entre niños infectados con cepas sensibles y resistentes a la penicilina y la cefalosporina.

\section{Material y métodos}

Este trabajo se realizó en el Hospital Infantil de México Federico Gómez, de marzo de 1994 a diciembre de 1998. Se trató de un estudio sin intervención, a partir del cual se dio seguimiento prospectivo a 38 niños con diagnóstico de meningitis bacteriana, hasta la resolución de los síntomas y signos clínicos. Los pacientes fueron incluidos en el protocolo tan pronto como se obtuvieron los resultados del cultivo del LCR, generalmente 48 horas después de la admisión. Unicamente se incluyó un aislamiento de cada paciente, independientemente de que se hubiese aislado el germen patógeno, de manera simultánea en LCR y en hemocultivo.

Todos los pacientes fueron evaluados desde su ingreso al servicio de urgencias por miembros del servicio de Infectología, quienes establecieron el tratamiento de inicio y alertaron al resto del personal médico para que no alteraran el esquema seleccionado. Los datos de evolución y mortalidad se obtuvieron al seguir a cada paciente durante su hospitalización y revisando el expediente clínico individual; asimismo, la evolución de los casos vivos se obtuvo por medio de los expedientes de consulta externa.

\section{A spectos clínicos}

Se hizo seguimiento a todos los pacientes, desde su ingreso al protocolo hasta su muerte o alta. En forma rutinaria se obtuvieron los siguientes datos: edad, sexo, evolución en días de la enfermedad, presencia de alguna enfermedad subyacente, prescripción de antimicrobianos en los últimos 15 días, adquisición de la infección actual en la comunidad o durante su estancia hospitalaria, condiciones clínicas al momento de la visita al servicio de urgencias, factores asociados a la infección como asistencia a guardería, contacto con pacientes con manifestaciones clínicas semejantes, otitis media aguda recurrente $\mathrm{u}$ otros factores.

\section{Definiciones}

Meningitis. Predominio de signos y síntomas neurológicos que incluyen rigidez de nuca, fontanela a ten- 
sión, cefalea, vómito, así como LCR característico de meningitis bacteriana, bacterioscopía con Gram, coaglutinación y cultivo positivo.

Fiebre. Se definió como elevación de la temperatura corporal en una o varias tomas en axila o recto con cifras $\geq 38.3^{\circ} \mathrm{C}$. La persistencia de este tipo de fiebre se interpretó como días febriles. Se consideró como resuelta cuando la temperatura permaneció por debajo de $38{ }^{\circ} \mathrm{C}$ por al menos 48 horas. La presencia de una nueva elevación térmica se consideró como fiebre secundaria.

Choque séptico. Cuando se presenta un paciente potencialmente infectado con colapso vascular, hipoperfusión periférica, disminución del gasto cardiaco, hipotensión arterial (presión sistólica $<65$ en lactantes y $<75$ en niños mayores), leucocitosis (más de 15 000/ $\mathrm{mm}^{3}$ con neutrofilia y bandemia), o bien, leucopenia (menos de $5000 / \mathrm{mm}^{3}$ ) o trombocitopenia con cuenta menor de $150000 / \mathrm{mm}^{3}$.

Enfermedad subyacente de importancia. Se consideró como un factor de riesgo presente, cuando el paciente tenía confirmado previamente un padecimiento de base como la enfermedad cardiaca congestiva con o sin insuficiencia (cardiomegalia, hepatoesplenomegalia y congestión o edema pulmonar), algún tipo de inmunosupresión (cáncer, lupus eritematoso sistémico, inmunodeficiencias primarias, cirrosis, síndrome nefrótico, síndrome de inmunodeficiencia adquirida), desnutrición, o bien, cuando el paciente recibía quimioterapia, radiaciones, esteroides o inmunomoduladores.

Infección nosocomial. Se definió como la presencia de signos y síntomas que se desarrollan por lo menos después del tercer día del internamiento y que no guardan relación con el padecimiento de base motivo de la hospitalización.

\section{Evaluación}

Los pacientes fueron evaluados todos los días anotando con precisión los signos y síntomas clínicos pertinentes. El estado de cada caso se revaluó los días 3 , 5 y 7 después de iniciado el tratamiento, y los hallazgos se compararon con los del ingreso y, al mismo tiempo, se categorizaron de la siguiente forma:

1. Sin cambio. Sin mejoría ni deterioro.

2. Mejoría. Cambio favorable del estado clínico pero sin completa resolución de los signos y síntomas.

3. Resolución. Paciente afebril por lo menos en las últimas 48 horas, con buena respuesta a los estímulos, alerta, con succión y deglución normal, sin presencia de signos meníngeos.
4. Desfavorable (falla). Pacientes sin cambio clínico o, incluso, con necesidad de cuidados especiales como apoyo ventilatorio, o bien, desarrollo de complicaciones inesperadas (convulsiones). En este rubro se incluyó a los enfermos en los que se alteró el esquema de tratamiento con base en la pobre respuesta clínica.

\section{Régimen terapéutico}

Para el análisis del esquema de tratamiento, los pacientes fueron divididos en los siguientes grupos:

- A. Pacientes que recibieron penicilina o un betalactámico equivalente (ampicilina), junto con cloranfenicol.

- B. Pacientes que recibieron ceftriaxona o cefotaxima.

- C. Pacientes que recibieron una cefalosporina de tercera generación combinada con vancomicina.

- D. Pacientes que recibieron exclusivamente vancomicina.

Cuando se decidió cambiar el esquema de tratamiento por pobre respuesta clínica, se realizó nueva punción raquídea antes de iniciar el o los nuevos antimicrobianos. Cuando el cambio se realizó al día 7 por buena respuesta, el caso se consideró como favorable. Las dosis diarias y los intervalos para cada fármaco utilizado se detallan a continuación: penicilina G, 100000 unidades/ $\mathrm{kg}$ divididas en dosis administradas cada cuatro horas por vía intravenosa (IV); ampicilina, $200 \mathrm{mg} / \mathrm{kg}$, cada seis horas por vía IV; cloranfenicol, $200 \mathrm{mg} / \mathrm{kg}$, cada seis horas por vía IV; ceftriaxona, $100 \mathrm{mg} / \mathrm{kg}$, cada 24 horas por vía IV; cefotaxima, $100 \mathrm{mg} / \mathrm{kg}$, cada seis horas por vía IV, y vancomicina, $40 \mathrm{mg} / \mathrm{kg}$, cada 12 horas por vía IV. La duración del tratamiento se prolongó durante 10 a 14 días en la mayoría de los pacientes.

\section{Estudio microbiológico (aislamientos)}

Las cepas de Streptococcus pneumoniae fueron identificadas por métodos convencionales estándar. ${ }^{13}$ Las cepas recién aisladas fueron probadas para conocer su susceptibilidad antimicrobiana preliminar, con el método de difusión en disco en placas de agar sangre con Mueller-Hinton. ${ }^{14}$ Se utilizó un disco impregnado con $1 \mathrm{mg}$ de oxacilina, a fin de reconocer aquellas cepas de neumococo con disminución de la sensibilidad a la penicilina; ulteriormente las cepas se conservaron en congelación. El escrutinio preliminar hecho a la penicilina con el disco de oxacilina (BBL, México) por el 
método de difusión de Kirby-Bauer se interpretó de la siguiente manera: una zona de inhibición $\geq 20 \mathrm{~mm}$ fue considerada como cepa sensible, mientras que aquellas cepas con una zona de inhibición $<20 \mathrm{~mm}$ se consideraron resistentes a la penicilina. ${ }^{15}$

\section{Serotipificación}

Se utilizó la reacción de hinchazón de la cápsula (reacción de Quellung), con sueros y factores (omniserum pools) obtenidos del Statens Seruminstitute, de Copenague, Dinamarca, para conocer los serotipos/serogrupos a los que pertenecía cada cepa.

\section{Antimicrobianos}

Todos los antimicrobianos fueron obtenidos en polvo, con calidad, potencia y fecha de caducidad de cada una de las empresas productoras, con el fin de preparar las soluciones para el abastecimiento. Los antimicrobianos utilizados fueron penicilina G potásica, ceftriaxona, ampicilina, cloranfenicol, eritromicina y vancomicina. Los resultados de la susceptibilidad fueron definidos de acuerdo con la guía de $1997^{16}$ del Comité Nacional de Estándares para el Laboratorio Clínico (NCCLS, por sus siglas en inglés).

La concentración mínima inhibitoria (CMI) se determinó por el método de microdilución, en medio de Mueller-Hinton (Difco, México), suplementado con 10 $\mathrm{mg} / \mathrm{l}$ de $\mathrm{MgCL}, 20 \mathrm{mg} / 1$ de $\mathrm{CaCl}^{2}$ y 5\% de sangre total desfibrinada de caballo, adicionando la cantidad apropiada de cada antimicrobiano. Los pozos de las microplacas se inocularon con $50 \mu$ de cada inóculo conteniendo $1 \times 10^{4}$ unidades formadoras de colonia. La lectura se realizó visualmente. Se utilizó como cepa de control a Streptococcus pneumoniae 49619 del American Type Culture Collection (ATCC). Se definió la CMI como la concentración más baja del antimicrobiano que impidió el crecimiento visible en un lapso de incubación de 18 a 24 horas a $35^{\circ} \mathrm{C}$. Los criterios de resistencia se ajustaron a los criterios del NCCLS ${ }^{15}$ de acuerdo con los siguientes puntos de corte en $\mu \mathrm{g} / \mathrm{ml}$ : penicilina (S) $\leq 0.06$, ( I ) 0.12-1.0, (R ) $\geq 2.0$; ceftriaxona y cefotaxima ( $\mathrm{S}) \leq 0.5$, ( I ) 1.0, ( R ) $\geq 2.0$; cloranfenicol (S) $\leq 4.0$, (R) $\geq 8.0$; eritromicina $(S) \leq 0.25$, ( I ) $0.5 \geq 1.0$, y vancomi$\operatorname{cina}(\mathrm{R}) \geq 1.0$.

\section{A nálisis estadístico}

La diferencia entre las proporciones se evaluó por medio de $\chi 2$ y la prueba exacta de Fisher cuando se consideró apropiado.

\section{Resultados}

\section{Características de los pacientes y presentación clínica}

Se completaron 38 cédulas individuales y estandarizadas para recoger la información de cada episodio de meningitis neumocócica, utilizando como fuente la información extractada del expediente clínico hospitalario correspondiente.

En el cuadro I se puede observar que la edad varió de 5 meses a 8 años: del total de pacientes, 24 (63\%) eran menores de dos años, y 14 eran mayores de esa edad $(37 \%) \square$; asimismo, 21 pacientes $(55 \%)$ eran del sexo masculino, y 17, del sexo femenino (45\%). La evolución clínica previa a su ingreso al hospital fue, en pro-

\section{Cuadro I}

Características demográficas y clínicas AL INGRESO DE 38 PACIENTES CON MENINGITIS neumocócica. Hospital Infantil de México Federico Gómez, 1994-1998

Aspecto

Frecuencia (\%)

Edad en meses ( varianza 5/12-8 años)

$\begin{array}{ll}\leq 24 \text { meses } & 24(63) \\ >24 \text { meses } & 14(37)\end{array}$

Sexo:

\begin{tabular}{cl} 
Masculino & $21(55)$ \\
\hline Femenino & $17(45)$
\end{tabular}

Duración (hrs.) de signología meníngea Previa al ingreso $(X \pm D E) \quad 58 \pm 32$

\begin{tabular}{ll} 
Padecimiento de base & $7(19)$ \\
\hline SIDA & 2 \\
\hline Hemato-oncológico & 2 \\
\hline Desnutrición & 2 \\
\hline Glomerulonefritis & 1
\end{tabular}

\begin{tabular}{lc} 
Antimicrobianos previos & $27(71)$ \\
\hline$\beta$-lactámicos & 19 \\
\hline Macrólidos & 8
\end{tabular}

\begin{tabular}{lc} 
Convulsiones previas al ingreso & $9(24)$ \\
\hline Generalizadas & 7 \\
\hline Focalizadas & 2
\end{tabular}


medio, de 58 horas, con variaciones de entre 1 y 4 días. Los de menor tiempo de evolución fueron niños con manifestaciones severas de daño meningoencefálico, en particular los que iniciaron sus signos y síntomas con crisis convulsivas.

Aproximadamente uno de cada 13 pacientes $(18.5 \%)$ que desarrollaron meningitis, tenía un padecimiento de base; en dos pacientes con SIDA y en uno con neuroblastoma, la infección se consideró como de adquisición nosocomial.

Más de 70\% de los pacientes habían recibido algún antimicrobiano en los últimos 15 días por vía oral; la mayoría recibió un betalactámico $(19 / 27)$ y los demás algún macrólido.

A su ingreso, nueve pacientes tenían antecedentes de haber convulsionado por lo menos en una ocasión, siete tuvieron crisis generalizadas y dos, focalizadas; cuatro pacientes (tres con crisis generalizada y uno con crisis focalizada) ingresaron en estado de coma y se consideraron igualmente con choque tóxico, de tal forma que requirieron apoyo ventilatorio.

\section{Sensibilidad antimicrobiana y serotipifi- cación}

Los serotipos / serogrupos 23F, 19F, 15, 6A, 6B, 14 y 11A representaron cerca de $70 \%$ de los serotipos predominantes $\square ; 27 / 38(71 \%)$ fuewn sensibles a penicilina e igualmente sensibles a macrólidos, cloranfenicol, cefalosporinas de tercera generación y vancomicina; $7 / 38$ se consideraron con sensibilidad intermedia $(18.4 \%)$, y únicamente $4 / 38$ se consideraron altamente resistentes a la penicilina (10.5\%). Una de estas cepas fue igualmente resistente a cefotaxima ( $\geq 8 \mu \mathrm{g} / \mathrm{ml}$ ). Cuando se agrupan las cepas con resistencia intermedia y elevada a la penicilina, se observa que la disminución de la sensibilidad a ese fármaco alcanza $29 \%$ (11/38); en cambio, no más de $2.3 \%$ fue resistente a la acción de las cefalosporinas de tercera generación (cefotaxima o ceftriaxona). La sensibilidad de las 38 cepas en contra de los fármacos probados se presenta en el cuadro II.

\section{Curso clínico y evolución}

En el cuadro III se resume el curso clínico y la evolución intrahospitalaria del grupo de estudio. Siete pacientes requirieron apoyo ventilatorio: cuatro de ellos lo necesitaron desde su ingreso, y en tres casos se indicó por presencia de periodos de apnea, cambios pupilares, signos de hipertensión endocraneana (como bradicardia) o por reinicio de crisis convulsivas. En general este apoyo ventilatorio se prolongó, en pro-

\begin{abstract}
Cuadro II
SUSCEPTIBILIDAD Y VALORES DE LA CONCENTRACIÓN MÍNIMA INHIBITORIA,* EN 38 CEPAS DE S. PNEUM ONIAE AISLADAS EN NIÑOS CON MENINGITIS. HOSPITAL Infantil de México Federico Gómez, 1994-1998
\end{abstract}

\begin{tabular}{|c|c|c|c|c|c|c|c|c|c|}
\hline \multirow[t]{2}{*}{ Antimicrobianos } & \multicolumn{3}{|c|}{ CMI $(\mu \mathrm{g} / \mathrm{ml})$} & \multicolumn{3}{|c|}{ No. de cepas } & \multicolumn{3}{|c|}{$\begin{array}{l}\text { Punto de corte } \\
\qquad(\mu \mathrm{g} / \mathrm{ml})\end{array}$} \\
\hline & Variación & $\mathrm{CMI}_{50}$ & $\mathrm{CMI}_{90}$ & $\mathrm{~S}$ & - & $R$ & $\mathrm{~S}$ & - & $R$ \\
\hline Penicilina & $0.015-4$ & 1 & 4 & 27 & 7 & 4 & $\leq 0.06$ & $0.1-$ & $\geq 2$ \\
\hline Cefotaxima & $0.008-2$ & 1 & 2 & 37 & 0 & 1 & $\leq 0.5$ & 1.0 & $\geq 2$ \\
\hline Cloranfenicol & $2-4$ & 2 & $\geq 8$ & 31 & 4 & 3 & $\leq 4.0$ & - & $\geq 8$ \\
\hline Macrólidos & $0.03>128$ & 1 & 128 & 27 & 4 & 7 & $\leq 0.25$ & 0.5 & $\geq 1.0$ \\
\hline Vancomicina & $0.25-0.5$ & 0.25 & 0.25 & 38 & 0 & 0 & - & - & $\geq 1.0$ \\
\hline
\end{tabular}

CMI: concentración mínima inhibitoria

* Referencias 15-17

medio, tres días. Las crisis convulsivas desarrolladas ya en el internamiento mostraron una tendencia a la focalización en seis de nueve pacientes; las demás fueron generalizadas. Aproximadamente $56 \%$ de los pacientes mostraron evolución favorable en los primeros tres días de hospitalización; la mediana de la fiebre fue de tres días y, en general, el estado febril tuvo un promedio de cinco. En 10 pacientes se presentó un nuevo evento febril, que inició entre el día 5 y 7 , acompañado por una reactivación de la signología neurológica; en nueve sujetos se realizó una tomografía computarizada, de tal forma que se pudo documentar la complicación. Cinco pacientes con derrame subdural fueron puncionados, de los cuales dos tenían empiema.

Todos los líquidos obtenidos fueron negativos a la bacterioscopía con tinción de Gram y al cultivo. En siete de los 17 pacientes que después de tres días de tratamiento no mostraron evolución favorable, se realizó una nueva punción lumbar, donde igualmente la bacterioscopía y el cultivo fueron negativos. Cuatro pacientes tenían poco cambio para el día 7 de evolución, y dos de ellos desarrollaron empiema subdural. En uno de elllos se había aislado del LCR el serotipo $23 \mathrm{~F}$ sensible a la penicilina, y en el otro se aisló el serotipo 6A igualmente sensible a la penicilina. En ambos casos se decidió cambiar el esquema de ampicilina-cloranfenicol por cefotaxima-vancomicina; la cefotaxima se suspendió a los 14 días y la vancomicina se siguió administrando sola hasta cumplir 25 días. En ningún caso se administró el régimen $\mathrm{D}$ (vancomicina sola) para iniciar el tratamiento de la meningitis.

La duración de la estancia hospitalaria presentó cifras extremas, desde siete días, como mínimo, hasta 78 días, como máximo, con una mediana de 12 y un promedio de 19 . 


\section{Cuadro III \\ Curso clínico y eVolución de 38 Niños Con MENINGITIS NeUmocócica. Hospital InfantIL DE México Federico Gómez, 1994-1998}

Aspecto

Frecuencia ( \% )

Intubación traqueal

$7(18)$

\begin{tabular}{ll} 
Convulsiones después de $72 \mathrm{hrs}$. & $9(24)$ \\
\hline Focales & 6 \\
\hline Generalizadas & 3
\end{tabular}

Duración de la fiebre (días)

\begin{tabular}{ll} 
Mediana & 3 \\
\hline Promedio \pm DE & $5 \pm 2.8$
\end{tabular}

\begin{tabular}{ll} 
Fiebre secundaria & $10(26)$ \\
\hline Punción lumbar después de $5^{\circ}$ día & $7(18)$ \\
\hline Bacterioscopía $(+)$ & 0 \\
\hline Cultivo $(+)$ & 0
\end{tabular}

\begin{tabular}{ll} 
Punción de higroma/empiema & $5(13)$ \\
\hline Bacterioscopía $(+)$ & 0 \\
\hline Cultivo $(+)$ & 0
\end{tabular}

\begin{tabular}{ll} 
Tomografía computarizada & $9(24)$ \\
\hline Infartos & $1 / 9$ \\
\hline Hidrocefalia & $2 / 9$ \\
\hline Derrame subdural & $5 / 9$ \\
\hline Empiema subdural & $2 / 9$ \\
\hline Cerebritis/Edema & $5 / 9$
\end{tabular}

Duración de la hospitalización (días)

\begin{tabular}{ll} 
Mediana & 12 \\
\hline Promedio $\pm \mathrm{DE}$ & $19 \pm 26$
\end{tabular}

Fallecimientos

Secuelas neurológicas

De los cinco pacientes que fallecieron en el grupo $(13 \%)$, dos tenían un padecimiento de base (SIDA y neuroblastoma); el primero cursaba con citopenias importantes, de tal forma que se aisló $S$. pneumoniae serotipo $23 \mathrm{~F}$ sensible a penicilina. El paciente falleció tres días después de iniciado el tratamiento con cefotaxima y vancomicina; este esquema de tratamiento se aplicó desde el inicio pues se trataba de un paciente de alto riesgo. Del paciente con cáncer se aisló el serotipo 15 altamente resistente a penicilina, eritromicina, cloranfenicol y cefalosporinas de tercera generación $(\geq 8$ $\mu \mathrm{g} / \mathrm{ml})$, pero sensible a la vancomicina. Puesto que se consideró desde su inicio como paciente de alto riesgo, recibió el esquema $C$ con cefotaxima y vancomicina. El paciente falleció a los cinco días de iniciado el tratamiento.

Por otro lado, tres pacientes con septicemia y componente meningoencefálico recibieron de inicio ampicilina y cloranfenicol; Uno de ellos llegó en estado de coma y con evidente choque tóxico, y falleció a las 24 horas de ingreso; no sobra decir que se le aisló el serotipo 12 sensible a penicilina. Dos pacientes, a quienes se les aisló el serotipo 19F sensible a todos los fármacos probados, falleciero: uno al tercer día y el otro al quinto día. El LCR tomado ocho horas antes del fallecimiento en el último paciente fue negativo a la bacterioscopía y en el cultivo. En el cuadro IV se resume la evolución clínica en función de la sensibilidad del S. pneumoniae a la penicilina y del régimen antimicrobiano recibido.

$\mathrm{Al}$ ser dados de alta, siete pacientes mostraban datos de secuelas: espasticidad (5), hemiparesis (1) e hidrocefalia comunicante (1). Se realizaron estudios audiológicos en todos los casos para valorar daño neurosensorial.

La presentación clínica y la severidad de la enfermedad fue similar en los niños con cepas penicilinosensibles que en aquellos que presentaban cepas penicilino-resistentes. Al comparar el riesgo de morir en relación con la presencia de cepas sensibles o resistentes no se encontró diferencia; en cambio, cuando se compararon otros factores de riesgo sólo el tener un

\section{Cuadro IV}

EvoluCión CLÍNICA DE LA MENINGITIS CAUSADA POR CEPAS DE S. PNEUM ONIAE SENSIBLES O RESISTENTES A LA PENICILINA, DE ACUERDO CON EL RÉGIMEN ANTIMICROBIANO RECIBIDO. HOSPITAL INFANTIL de México Federico Gómez, 1994-1998

\begin{tabular}{|c|c|c|c|c|c|c|c|}
\hline \multirow[t]{2}{*}{ Día } & \multirow[t]{2}{*}{ Grupo* } & \multicolumn{3}{|c|}{ PEN-sensible $\mathrm{n}=27$} & \multicolumn{3}{|c|}{ PEN - resistente ${ }^{\ddagger} n=11$} \\
\hline & & $F$ & NOF & M & $F$ & $\mathrm{No}$ & M \\
\hline \multirow[t]{3}{*}{3} & $A$ & 9 & 3 & 3 & 3 & 2 & 0 \\
\hline & B & 4 & 6 & 0 & 2 & 3 & 0 \\
\hline & C & 3 & 2 & 1 & 0 & 1 & 1 \\
\hline \multirow[t]{3}{*}{7} & A & 11 & 1 & 0 & 6 & 0 & 0 \\
\hline & $B$ & 8 & 2 & 0 & 4 & 1 & 0 \\
\hline & $C$ & 4 & 0 & 0 & 0 & 0 & 0 \\
\hline
\end{tabular}

* Grupo de tratamiento: $\mathrm{A}=$ =ampicilina más cloranfenicol; $\mathrm{B}=$ cefatoxima 0
ceftriaxona; $\mathrm{C}=$ cefotaxima 0 ceftriaxona más vancomicina ₹ $\mathrm{F}=$ Favorable; $\mathrm{N}$ oF $=\mathrm{N}$ o favorable; $\mathrm{M}=$ muertos

salud pública de méxico / vol.41, no.5, septiembre-octubre de 1999 
padecimiento de base previo, mostró $p<0.001$ la prueba exacta de Fisher.

\section{Discusión}

La resistencia a la penicilina y a otros betalactámicos, e incluso la multirresistencia a diversos fármacos entre las cepas de S. pneumoniae, están ampliamente diseminadas en todo el mundo y con una tendencia a seguir aumentando en forma desproporcionada. ${ }^{18-20} \mathrm{En}$ nuestro país este problema, se está acrecentando desde 1981.21,22 Por ahora la atención de las infecciones producidas por el S. pneumoniae se centra en varios aspectos que requieren de una solución a corto plazo; el primero se refiere al tratamiento apropiado de las infecciones neumocócicas sistémicas, fundamentalmente de las del sistema nervioso central; el segundo está en función de la magnitud de la resistencia, para alterar la evolución clínica de las infecciones invasivas; el tercero está relacionado con la identificación de los factores de riesgo que intervienen en la propensión a las infecciones neumocócicas, así como del curso de la evolución clínica y, finalmente, el cuarto implica definir la prevalencia de serotipos/serogrupos en las diferentes comunidades, de tal forma que se pueda identificar con cierta precisión la fórmula de la vacuna neumocócica que requiere la población en riesgo.

En el presente estudio la resistencia intermedia a la penicilina fue de $18.4 \%$, y la elevada, de $10.5 \%$ (en total de $28.9 \%$ ). En cambio, la resistencia elevada a las cefalosporinas de tercera generación únicamente fue de 2.6\%; al cloranfenicol, de 7.8\%, y a los macrólidos, de $18.4 \%$. Estas cifras se ubican entre las más bajas de la literatura inglesa ${ }^{19,20,23-25}$ y las más altas de la europea ${ }^{26,27}$ y la asiática. ${ }^{28-30}$

En la literatura publicada ${ }^{9,22-30}$ con propósitos semejantes se observó que, en general, la evolución clínica de los pacientes con infecciones neumocócicas invasivas y, en particular, con meningitis, es muy semejante independientemente de que la cepa de $S$. pneumoniae sea sensible o resistente a la penicilina $\mathrm{u}$ otros betalactámicos. En esta investigación se observó que la presentación clínica, la severidad de la enfermedad, la morbilidad y la mortalidad, así como las estancias hospitalarias, fueron similares entre los niños infectados con cepas sensibles o resistentes a la penicilina. Sin embargo, con la presencia de un solo caso, donde la resistencia fue elevada a la cefotaxima, no es posible concluir que tal diferencia no existe, o bien, que puede llegar a existir si la muestra de estudio es mayor. Lo que sí es un hecho que llama la atención, es que en la meningitis son menos frecuentes las cepas con resistencia elevada a la penicilina, tal como ha sido notificado en otros casos, ${ }^{23}$ ello, desde un punto de vista práctico, concuerda con la evolución que mostró el grupo de pacientes en estudio, pues realmente no se demostró diferencia estadística al comparar el esquema de tratamiento A (ampicilina-cloranfenicol) contra el B (cefotaxima o ceftriaxona); sin embargo, con estos mismos resultados, se puede comentar que el uso del esquema $\mathrm{C}$ (cefotaxima-vancomicina) no tuvo ninguna justificación, independientemente de que se hubiese considerado a los pacientes como de alto riesgo o de gravedad extrema. En ningún caso se demostró que la resistencia bacteriana de alguna de las cepas fuese la responsable de alterar la evolución o, quizá más importante, de algún fallecimiento. Como una consecuencia práctica, en un hospital de concentración como el de este estudio-, se justifica que se aplique cefotaxima o ceftriaxona en niños con meningitis neumocócica, como tratamiento empírico inicial, y únicamente se justificaría la asociación con vancomicina si se demuestra que la evolución clínica no es favorable y si se conoce el perfil de sensibilidad de la cepa aislada. Es interesante señalar que la práctica de una segunda punción lumbar no se justifica cuando se tiene una evolución aceptable y cuando no hay signos y síntomas neurológicos de progresión del daño.

La proporción de cepas de S. pneumoniae resistente a otros fármacos diferentes a los betalactámicos es variable; en el cuadro II se observa que el cloranfenicol puede ser eficaz para el tratamiento de la meningitis neumocócica en más de 90\%. La resistencia no ha tenido tendencia a la progresión; en algunos países americanos como Estados Unidos de América, la resistencia es cercana a $4 \%{ }^{20}$ En cambio, en Sudáfrica las cifras son las más altas. ${ }^{31}$ Respecto a la eritromicina y sus congéneres, la resistencia sí tiende a incrementarse progresivamente, y ello está en relación directa con su cada vez más indiscriminada administración por vía oral para procesos infecciosos con indicaciones muy cuestionables. Las cifras americanas ${ }^{20}$ son de aproximadamente $12 \%$, y en México son cercanas al 30\%, cifra que equivale al doble de lo notificado hace cinco años. ${ }^{22}$

La evolución clínica se ve comprometida, más que por la presencia de cepas resistentes a los fármacos antimicrobianos administrados al paciente, por una serie de factores de riesgo que son de tomar en cuenta en el análisis individual de cada enfermo. El antecedente de administración previa de antibióticos es un factor que se presenta en más de $70 \%$ de los casos, y en su mayoría los reciben por vía oral en cefalosporinas y macrólidos. La presencia de un padecimiento de base es crítico en la evolución dentro de los primeros tres a cinco días, y el riesgo de morir bajo esas circunstancias es mayor $(p<0.001)$. 
Los serotipos / serogrupos de las cepas de S. pneumoniae involucrados son muy semejantes (aproximadamente $70 \%$ ) a los observados en otros estudios de meningitis neumocócica. Estos mismos serotipos se encuentran incluidos casi en su totalidad en la vacuna de 23 polisacáridos, la cual no tiene una buena respuesta precisamente en los niños menores de dos años de edad. La prevención de la enfermedad neumocócica invasiva, por medio de una vacuna conjugada que incluya los serotipos más frecuentes, muy pronto será posible..$^{32}$ Mientras tanto es necesario continuar con la vigilancia epidemiológica de los patrones de resistencia bacteriana del neumococo.

\section{Referencias}

1.Zangwill KM,Valdheim CM,Vannier AM, Hemenway LS, G reenberg DP, $W$ ard JI. Epidemiology of invasive pneumococcal diseases in Southern $C$ alifornia: Implications for the design and conduct of a pneumococcal conjugated vaccine efficacy trial.J Infect $D$ is 1996;174:752-759.

2. Kronenberg C B, Hoffman RE, Lezotte DC, Marine W M. Invasive penicillin-resistant pneumococcal infections: A prevalence and historical cohort study. Emergin Infect $D$ is 1996;2:121-124.

3. Butler JC, H offman J,C etron MS, Elliot JA, Facklam RR, Brosman RF et al. The continental emergence of drug-resistant Streptococcus pneumoniae in the United States:A n updating from the Centers for D isease Control and Prevention's Pneumococcal Sentinel Surveillance System.J Infect D is 1996; 174:986-993.

4. De Velasco EA, Verheul FM, Verhoef J, Snippe H. Streptococcus pneumoniae: Virulence factors, pathogenesis, and vaccines. Microbiol Rev 1995; 59:591-603

5. Klugman KP. Pneumococcal resistance and antibiotics. Clin Microbiol Rev 1990; 3:171-196.

6. Tomasz A . Antibiotic resistance in Streptococcus pneumoniae. C lin Infect $D$ is 1997; 24 suppl 1:85-88.

7. C ampbell D, Silberman R. D rug-resistant Streptococcus pneumoniae. C lin Infect Dis 1998;26:1188-1195.

8. Friedland IR, Istre GR. Management of penicillin-resistant pneumococcal infections. Pediatr Infect D is J 1992;11:433-435.

9.Tan TQ , Mason EO , Kaplan SL. Systemic infections due to a Streptococcus pneumoniae relatively resistant to penicillin in a childrens hospital: Clinical management and outcome. Pediatrics 1992;90:928-933.

10. Friedland IR, MCC racken GH Jr. Management of infections caused by antibiotic resistant Streptococcus pneumoniae. N Engl J Med 1994;331: 377-382.

11. A merican A cademy of Pediatrics: C ommittee on Infectious D iseases. Therapy for children with invasive pneumococcal infections. Pediatrics 1997:99:289-299.

12. Lutsar I, McC racken GH Jr, Friedland IR. Antibiotic pharmacodynamics in cerebrospinal fluid. C lin Infect D is 1988;27:1117-1129.

13. Ruoff KL,W hiley RA, Beighton D. Streptococcus. En: Murray RR, Baron EJ, Pfaller MA,Tenover FC, Yolken RH, ed. Manual of clinical microbiology. 7a. edición.W ashington, D.C.:ASM Press, 1999:283-296.

14. Jacobs MR, Gaspar MN, Robin-Browner RM, Koornhof HJ.Antimicrobial susceptibility testing of pneumococci. 2. Determination of optimal disk diffusion test for detection of penicillin $G$ resistance. J Antimicrob Chemother 1980;6:53-64.
15. N ational Committee for Clinical Laboratory Standards. Performance standards for antimicrobial susceptibility testing. Sixth informational suplement M1150-56. W ayne, PA: N ational Committee for Clinical Laboratory Standards, 1995.

16. N ational C ommittee for C linical Laboratory Standards. Minimum inhibitory concentrations (MIC) Interpretative standards ( $\mu \mathrm{g} / \mathrm{ml})$ for Streptococcus spp.,Table 2C.M 100-S7.W ayne, PA:N ational Committee for Clinical Laboratory Standards, 1997;17(2).

17. A ppelbaum PC. Antimicrobial resistance in Streptococcus pneumoniae: An overview. C lin Infect D is 1992;15:77-83.

18. Friedland IR. Comparison of the response to antimicrobial therapy of penicillin-resistant and penicillin susceptible pneumococcal disease. Pediatr infect D is J 1995;14:885-890.

19. D oern TG V, Pfaller MA, Kugler K, Freeman J, Jones RN . Prevalence of antimicrobial resistance among respiratory tract isolates of Streptococcus pneumoniae in N orth America: 1997 results from SEN TRY Antimicrobial Surveillance Program. Clin Infect D is 1998; 27:764-770.

20. Mufson MA. Editorial response: Penicillin-resistant Streptococcus pneumoniae increasingly threatens the patient and challenges the physician. C lin Infect $D$ is 1998;27:771-773.

21. G uiscafré GH, García MM, Trejo PA, G arcía M, Hernández VR, Muñoz $H O$. Frecuencia de Haemophilus influenzae resistente a ampicilina y de Streptococcus pneumoniae resistente a penicilina en portadores sanos.Arch Invest Med Mex 1981;12:141-151.

22. Calderón JE, Echániz AG, C onde-G lez CJ, Rivera SR, BarrigaAG, Solórzano SF et al. Resistencia y serotipificación de 83 cepas de Streptococcus pneumoniae aisladas de niños portadores asintomáticos y enfermos. Bol Med Hosp Infant Mex 1993;50:854-859.

23. Deeks SL, Palacio R, Ruvinsky R, Kertesz DA, Hortal M, Rossi A et al. Risk factors and course of illness among children with invasive penicillinresistant Streptococcus pneumoniae. Pediatrics 1999;103:409-413.

24. Arditi M, Mason EO, Bradley JS, Tan TQ, Barson W J, Schutze GE et al. Three-year multicenter surveillance of pneumococcal meningitis in children: Clinical characteristics, and outcome related to penicillin susceptibility and dexamethasone use. Pediatrics 1998;102:1087-1096.

25. Kaplan SL, Mason EO, Barson W J, W ald ER, Arditi M, Tan TQ et al. Three-year multicenter surveillance of systemic pneumococcal infections in children. Pediatrics 1998;102:538-545.

26. Pallares R, Liñares J,Vadillo M, C abellos C, Manresa F,Viladrich PF et al. Resistance to penicillin and cephalosporin and mortality from severe pneumococcal pneumonia in Barcelona, Spain. N Engl J Med 1995;333: 474-480.

27. García-Leoni $M E$, Cercenado $E$, Rodeño $P$, Bernaldo de $Q$ uirós $C L$, Martínez-Hernández D, Bouza E. Susceptibility of Streptococcus pneumoniae to penicillin: A prospective microbiological and clinical study. Clin Infect Dis 1992,14:427-435.

28. Chiou CC, Liu YC, HuangTS, H wang W K, W ang JA, Lin HH, et al. Extremely high prevalence of naso pharyngeal carriage of penicillin-resistant Streptococcus pneumoniae among children in Kaohsiung,Taiwan.J C lin Microbiol 1998;36:1933-1937

29. Lee HJ, Park JY, Jang SH, Kim JH, Kim EC, C hoi KW. High incidence of resistance to multiple antimicrobials in clinical isolates of Streptococcus pneumoniae from a University Hospital in Korea. Clin Infect D is 1995; 20:826-835.

30. Choi EH, Lee HJ. Clinical outcome of invasive infections by penicillinresistant Streptococcus pneumoniae in Korean children. Clin Infect dis 1998;26:1346-1354

31. Friedland IR, Klugman KP. Failure of chloramphenicol therapy in penicillin-resistant pneumococcal meningitis. Lancet 1992;339:405-408.

32. Rennels MB, Edwards KM, Keyserling HL, Reisinger KS, Hogerman DA, Madore DV et al. Safety and immunogenicity of heptavalent pneumococcal vaccine conjugated to $\mathrm{CRM}_{197}$ in United States infants. Pediatrics 1998;101:604-609. 